\title{
Adubação orgânica e Lithothamnium no cultivo da pitaia vermelha
}

\section{Organic fertilizer and Lithothamnium on the cultivation of red pitaia}

\author{
Ana Claudia Costa ${ }^{1 *}$; José Darlan Ramos ${ }^{2}$; Fábio Oseias dos Reis Silva ${ }^{3}$; \\ Thatiane Padilha de Menezes²; Rodrigo Amato Moreira ${ }^{4}$; Mariene Helena Duarte ${ }^{5}$
}

\section{Resumo}

\begin{abstract}
A pitaia vermelha [Hylocereus undatus (Haw.) Britton \& Rose] é uma cactácea frutífera nativa das florestas tropicais do México e das Américas Central e do Sul que tem demonstrado grande potencial nos mercados interno e externo, devido a sua rusticidade e aparência exótica. O trabalho foi realizado com o objetivo de avaliar a influência da adubação orgânica e a aplicação de Lithothamnium (granulado bioclástico) na produção e qualidade de frutos de pitaia vermelha. O delineamento experimental foi em blocos casualizados com oito tratamentos: controle (sem adubação), esterco bovino (EB), esterco de galinha (EG), granulado bioclástico (GB), esterco bovino + esterco de galinha, esterco bovino + granulado bioclástico, esterco de galinha + granulado bioclástico e esterco bovino + esterco de galinha + granulado bioclástico, aplicados a cada três meses desde setembro de 2008, com três blocos, e a parcela experimental composta por quatro plantas. As análises feitas foram: produtividade, número de frutos por planta, massa de fruto, rendimento de polpa, diâmetro transversal, diâmetro longitudinal, espessura de casca, teor de sólidos solúveis (SS), acidez titulável (AT), pH e relação SS/AT. As maiores produtividades foram obtidas nos tratamentos contendo EB + EG + GB (7,16 t ha $\left.{ }^{-1}\right)$ e EB + EG $(6,90 \mathrm{t}$ ha $\left.^{-1}\right)$ em 2011, sendo que o primeiro tratamento também apresentou maior produtividade em $2012(9,40$ $\left.\mathrm{t} \mathrm{ha}^{-1}\right)$. Maior número de frutos por planta foi obtido quando se realizou a adubação com EB $+\mathrm{EG}+\mathrm{GB}$ nos dois anos avaliados. Não houve, no entanto, diferença estatística entre as características químicas dos frutos de pitaia com diferentes adubações.
\end{abstract}

Palavras-chave: Hylocereus undatus, granulado bioclástico, produção, qualidade de frutos

\begin{abstract}
The red pitaya is a fruit cactus native from the rainforests of Mexico, Central America and South America that has shown great potential in domestic and foreign markets, due to its hardiness and exotic appearance. The work was carried out to evaluate the influence of organic fertilizers and the application of Lithothamnium (calcified seaweed) on production and fruit quality of red pitaia [Hylocereus undatus (Haw.) Britton \& Rose]. The experimental design was a randomized block with eight treatments: control (no fertilization), cattle manure $(\mathrm{CaM})$, chicken manure $(\mathrm{ChM})$, calcified seaweed $(\mathrm{CS})$, cattle manure + chicken manure, cattle manure + calcified seaweed, chicken manure + calcified seaweed and cattle manure + chicken manure + calcified seaweed. The fertilizers were applied every three months since September 2008, with three blocks, and the experimental unit consisted of four plants. The analyzes done were: yield, number of fruits per plant, fruit mass, pulp yield, transverse diameter, longitudinal

${ }^{1}$ Eng $^{\mathrm{a}}$ Agr $^{\mathrm{a}}$, Prof ${ }^{\mathrm{a}}$ Dr $^{\mathrm{a}}$ em Agronomia, Universidade do Estado de Mato Grosso, UNEMAT, Nova Xavantina, MT. E-mail: anaclaudiacosta87@hotmail.com

2 Eng ${ }^{\text {os }}$ Agr $^{\text {os }}$, Profs. Drs. em Agronomia, Universidade Federal de Lavras, UFLA, Lavras, MG. E-mail: darlan@dag.ufla.br; thatiagro@yahoo.com.br

3 Eng $^{\circ}$ Agr $^{\circ}$, Discente do Curso de Doutorado em Agronomia, UFLA, Lavras, MG. E-mail: foseias@yahoo.com.br

4 Eng $^{\mathrm{O}}$ Agr $^{\mathrm{o}}$, Dr. em Agronomia, Universidade Federal dos Vales do Jequitinhonha e Mucuri, UFVJM, Diamantina, MG. E-mail: amatomoreira@yahoo.com.br

5 Licenciada em Química, Discente de Doutorado em Química, UFLA, Lavras, MG. E-mail: marieneduarte@hotmail.com

* Autor para correspondência
\end{abstract}


diameter, peel thickness, soluble solids (SS), titratable acidity (TA), $\mathrm{pH}$ and SS/TA ratio. The highest productivities were obtained in treatments containing $\mathrm{CaM}+\mathrm{ChM}+\mathrm{CS}\left(7.16 \mathrm{tha}^{-1}\right)$ and $\mathrm{CaM}+\mathrm{ChM}$ $\left(6.90 \mathrm{t} \mathrm{ha}^{-1}\right)$ in 2011, and the first treatment also showed higher productivity in $2012\left(9.40 \mathrm{t} \mathrm{ha}^{-1}\right)$. Greater number of fruits per plant was obtained when fertilization was carried out with $\mathrm{CaM}+\mathrm{ChM}+\mathrm{CS}$ in the two years evaluated. There were no significant differences among the chemical characteristics of red pitaia fruits with different fertilizations.

Key words: Hylocereus undatus, calcified seaweed, yield, fruit quality

\section{Introdução}

O consumo de frutas no Brasil tem aumentado nos últimos anos (SILVEIRA et al., 2011). Essa tendência está ligada ao aumento do poder aquisitivo e também pela conscientização da população. Apesar do consumo se concentrar nas frutas tradicionais, algumas frutas exóticas veem se destacando. Dentre essas pode ser citada a pitaia vermelha, cactácea nativa das florestas tropicais do México e das Américas Central e do Sul (HERNÁNDEZ, 2000).

Um dos problemas que influenciam o cultivo da pitaia é a falta de informações a respeito do manejo nutricional. Atualmente, são aplicadas doses empíricas, baseadas na experiência dos cultivos, ou se utilizam níveis recomendados para outros países, com sistemas ecológicos diferentes das regiões produtoras brasileiras (CAVALCANTE et al., 2011). O uso de adubos orgânicos pode ser uma alternativa promissora para o desenvolvimento da cultura, pois melhora a agregação do solo, especialmente porque influencia positivamente a infiltração e a capacidade de retenção de água, bem como a drenagem, aeração, temperatura e penetração de raízes (OLIVEIRA et al., 2009).

Os benefícios dos fertilizantes orgânicos foram estudados por Santos e Mendonça (2000), concluindo que há melhorias nas propriedades físicas do solo a partir da redução da densidade, bem como liberação de ácidos orgânicos, como os ácidos húmicos e fúlvicos, ácidos orgânicos de baixa massa molecular, ácido tânico, aminas, dentre outros. Galbiatti et al. (1996) e Moreira et al. (2010) reportaram enriquecimento dos atributos químicos do solo na capacidade de retenção de bases promovido pela aplicação do fertilizante orgânico.
Para os autores, esse aspecto exerce relevância, visto que a obtenção de elevado rendimento com qualidade de frutos está diretamente associada a uma nutrição balanceada da cultura, como observaram Oliveira e Caldas (2004) e Damatto Júnior, Leonel e Pedroso (2005) para o mamoeiro (Carica papaya L.) e maracujazeiro-doce (Passiflora alata Curtis), respectivamente. Adicionalmente, deve-se registrar que uma planta bem suprida tem maior tolerância à incidência de pragas e doenças.

Os corretivos de solo são necessários para iniciar o processo de agricultura orgânica no Brasil, sendo os mais utilizados o calcário dolomítico, calcítico e magnesiano. Existem outros insumos agrícolas, como calcário de conchas, que também podem ser empregados como corretivos, mas são pouco utilizados (MARQUES, 2010).

Os granulados bioclásticos, são algas calcárias (Lithothamnium) que contribuem para o melhoramento físico, químico e biológico do solo, corrigindo a acidez, aumentando a disponibilidade dos fertilizantes para as plantas e a atividade biológica do solo, nos quais favorecem a disponibilidade do fósforo e ativam o desenvolvimento das bactérias autotróficas responsáveis pela nitrificação (DIAS, 2000). Trabalhos com granulados bioclásticos demonstraram correção da acidez do solo (MELO; FURTINI NETO, 2003) e melhor desenvolvimento de mudas de mamoeiro (HAFLE et al., 2009).

Diante do exposto, o trabalho foi realizado com o objetivo de avaliar a influência da adubação orgânica e a aplicação de corretivo de solo (granulado bioclástico) na produção e qualidade de frutos de pitaia vermelha. 


\section{Material e Métodos}

O trabalho foi realizado no Setor de Fruticultura do Departamento de Agricultura (DAG) da Universidade Federal de Lavras (UFLA), LavrasMG, de junho de 2010 a maio de 2012. O município está localizado a $21^{\circ} 14^{\prime} 06^{\prime \prime}$ de latitude Sul, $45^{\circ} 00^{\prime} 00^{\prime \prime}$ de longitude Oeste e altitude média de $918 \mathrm{~m}$. O clima da região é do tipo $\mathrm{Cwb}$, temperado chuvoso (mesotérmico), segundo a classificação de Köeppen. Os dados de temperatura, umidade e precipitação do período avaliado estão apresentados na Figura 1.

Figura 1. Temperaturas máxima, mínima e média, umidade e precipitação para os meses de junho de 2010 a maio de 2012.

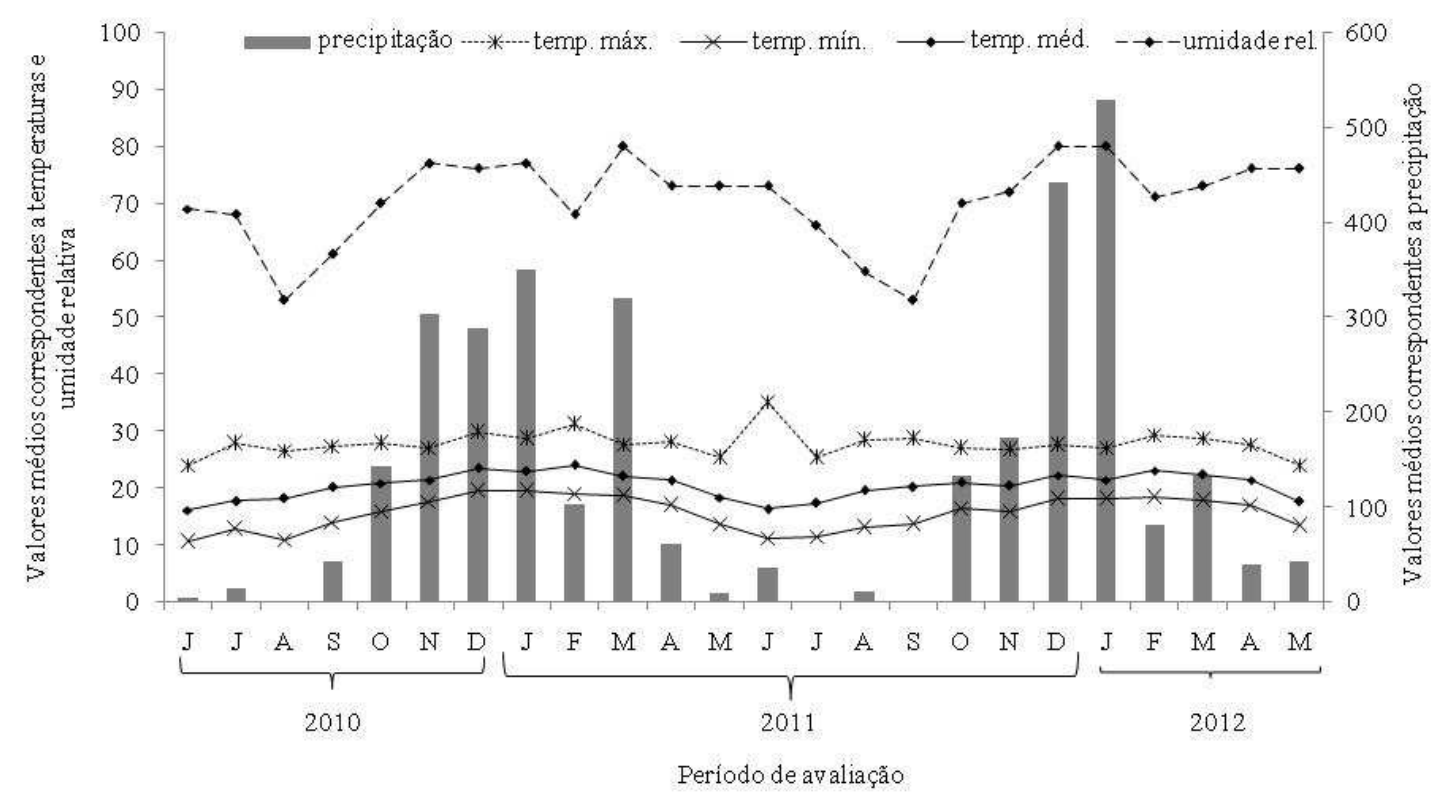

Fonte: Elaboração dos autores.

As mudas de pitaia vermelha foram plantadas em covas de $0,5 \times 0,5 \times 0,5 \mathrm{~m}$, no espaçamento de 3 $\mathrm{m} \times 3 \mathrm{~m}$ (densidade de 1.111 plantas $\mathrm{ha}^{-1}$ ) em junho de 2008. As covas foram adubadas com fósforo (300 g de superfosfato simples, $20 \%$ de $\mathrm{P}_{2} \mathrm{O}_{5}$ ) e $20 \mathrm{~L}$ de esterco bovino no plantio e os tratamentos começaram a ser aplicados três meses após a implantação da cultura. As análises do esterco bovino, de galinha e do granulado bioclástico utilizados no experimento estão apresentadas na Tabela 1.
Após o plantio, as mudas foram tutoradas em mourões de eucalipto perpendiculares ao solo até alcançar a altura de $1,8 \mathrm{~m}$. A condução foi feita através de desbrotas, deixando a planta em haste única até alcançar a latada formada por bambu acima dos mourões para sustentação dos cladódios. Foi feita análise de solo antes da implantação do experimento nas profundidades de $0-20 \mathrm{~cm}$ e de 21 $40 \mathrm{~cm}$ (Tabela 2). 
Tabela 1. Análise química do esterco bovino (EB), esterco de galinha (EG) e granulado bioclástico (GB), aplicados nas plantas de pitaia vermelha.

\begin{tabular}{|c|c|c|c|c|c|c|c|c|c|c|c|}
\hline Adubo & $\mathrm{N}$ (total) & $\mathrm{P}$ & $\mathrm{K}$ & $\mathrm{Ca}$ & $\mathrm{Mg}$ & $\mathrm{S}$ & B & $\mathrm{Cu}$ & $\mathrm{Fe}$ & $\mathrm{Mn}$ & $\mathrm{Zn}$ \\
\hline & $\%$ & & & $-\mathrm{g} \mathrm{kg}$ & - & & \multicolumn{5}{|c|}{---------------mg kg } \\
\hline${ }^{(1)} \mathrm{EB}$ & 1,6 & 5,7 & 15,5 & 100 & 3,3 & 1,2 & 19 & 32 & - & 220 & 135 \\
\hline${ }^{(2)} \mathrm{EG}$ & 3,4 & 19,5 & 24,3 & 100 & 5,7 & 3,0 & 21,8 & 65 & 1189 & 378 & 292 \\
\hline${ }^{(3)} \mathrm{GB}$ & - & 0,78 & 6,9 & 551 & 48 & 3,79 & 38,2 & 6,5 & 5576 & 443 & 15,4 \\
\hline
\end{tabular}

${ }^{(1)} \mathrm{EB}=$ Esterco bovino ${ }^{(2)} \mathrm{EG}=$ Esterco de galinha ${ }^{(3)} \mathrm{GB}=$ Granulado bioclástico.

Fonte: Elaboração dos autores.

Tabela 2. Análise de solo da área experimental de pitaia vermelha nas profundidades (Prof.) de 0-20 cm e de 21-40 $\mathrm{cm}$.

\begin{tabular}{|c|c|c|c|c|c|c|c|c|c|c|}
\hline Prof. & $\mathrm{pH}$ & $\mathrm{P}$ & K & $\mathrm{Ca}$ & $\mathrm{Mg}$ & $\mathrm{Al}$ & $\mathrm{H}+\mathrm{Al}$ & SB & $\mathrm{t}$ & CTC \\
\hline & \multicolumn{4}{|c|}{---mg dm${ }^{-3}---$} & & 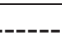 & $-----\mathrm{cmc}$ & $\mathrm{Im}^{-3}-$ & - & \\
\hline $0-20$ & 6,0 & 8,5 & 36 & 3 & 0,5 & 0,1 & 2,6 & 3,6 & 3,7 & 6,2 \\
\hline \multirow[t]{2}{*}{$21-40$} & 6,1 & 2,5 & 17 & 2,2 & 0,2 & 0,1 & 2,6 & 2,4 & 2,5 & 2,5 \\
\hline & \multicolumn{10}{|c|}{ 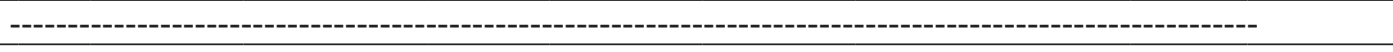 } \\
\hline \multirow[t]{2}{*}{ Prof. } & $\mathrm{m}$ & $\mathrm{V}$ & $\mathrm{MO}$ & $\mathrm{B}$ & $\mathrm{Zn}$ & $\mathrm{Cu}$ & $\mathrm{Fe}$ & $\mathrm{Mn}$ & $\mathrm{S}$ & Prem \\
\hline & $\%$ & $\%$ & dag $\mathrm{kg}^{-1}$ & \multicolumn{6}{|c|}{ - } & $\mathrm{mg} \mathrm{L}^{-1}$ \\
\hline $0-20$ & 3 & 58 & 2,4 & 0,2 & 5 & 4,6 & 79,2 & 28,3 & 5,8 & 27,2 \\
\hline $21-40$ & 4 & 48,4 & 1,6 & 0,2 & 1 & 2,7 & 16,5 & 16,1 & 5,8 & 26,4 \\
\hline
\end{tabular}

Fonte: Elaboração dos autores.

O delineamento experimental utilizado foi em blocos casualizados com oito tratamentos, três blocos e a parcela experimental composta por quatro plantas. Os tratamentos consistiram em: 1controle (sem adubação); 2- esterco bovino (14 kg); 3- esterco de galinha ( $2 \mathrm{~kg})$; 4- granulado bioclástico $(35 \mathrm{~g}) ; 5$ - esterco bovino + esterco de galinha (14 kg de $\mathrm{EB}+2 \mathrm{~kg}$ de EG); 6- esterco bovino + granulado bioclástico (14 kg de EB + 35 g de GB); 7- esterco de galinha + granulado bioclástico $(2 \mathrm{~kg}$ de $\mathrm{EG}+$ $35 \mathrm{~g}$ de GB) e 8-esterco bovino + esterco de galinha + granulado bioclástico (14 kg de EB + $2 \mathrm{~kg}$ de $\mathrm{EG}+35 \mathrm{~g}$ de GB). De acordo com os tratamentos, aplicou-se $14 \mathrm{~kg}$ de esterco bovino, $2 \mathrm{~kg}$ de esterco de galinha e $35 \mathrm{~g}$ de granulado bioclástico por planta. As adubações foram realizadas a cada três meses a partir de setembro de 2008.

Os frutos foram colhidos de dezembro de 2010 a maio de 2011 e de dezembro de 2011 a maio de 2012. Foi feita a contagem número de frutos por planta e a pesagem desses frutos para calcular a produção (número de frutos por planta) e a produtividade por planta que foi então estimada por hectare (produtividade). As avaliações físicoquímicas foram realizadas no mês de janeiro de 2011 e 2012. A determinação do ponto de colheita foi baseada na coloração vermelha da casca. As avaliações feitas foram: produtividade $\left(\mathrm{t} \mathrm{ha} \mathrm{h}^{-1}\right)$, número de frutos por planta, massa $(\mathrm{g})$, rendimento de polpa $(\%)$, espessura de casca $(\mathrm{mm})$, diâmetro transversal (mm), diâmetro longitudinal ( $\mathrm{mm}), \mathrm{pH}$, acidez titulável (\%), teor de sólidos solúveis ( ${ }^{\circ}$ Brix) e relação SS/AT.

Para determinação da massa dos frutos, utilizouse uma balança semi-analítica; os diâmetros longitudinal e transversal dos frutos, foram medidos com auxílio de paquímetro digital; o rendimento de polpa, foi estimado separando-se a casca da polpa e pesando-se apenas a polpa; a espessura da casca, foi mensurada com auxílio de paquímetro digital; o teor de sólidos solúveis (SS), foi determinado com refratômetro manual, sendo os resultados expressos 
em graus Brix (AOAC, 2007); o $\mathrm{pH}$, foi medido após a mistura de 1,0 g de polpa com $20 \mathrm{~mL}$ de água; a acidez titulável, foi estimada pesando-se 1,0 g da polpa, juntando-se em $20 \mathrm{~mL}$ de água, que, depois de misturados foram titulados com $\mathrm{NaOH}$ a $0,1 \mathrm{~mol} \mathrm{~L}^{-1}$, tendo como indicador fenolftaleína a $0,1 \%$. Os resultados foram expressos em gramas de ácido málico por $100 \mathrm{~g}$ de polpa (AOAC, 2007). A relação SS/AT foi obtida pela razão entre os teores de sólidos solúveis (SS) e a acidez titulável (AT).

Em setembro de 2011 foram retirados cladódios das plantas para a realização da análise dos teores de nutrientes contidos na matéria seca. Foram utilizados cladódios com 12 meses de idade, ou seja, provenientes da primavera do ano anterior, padronizando-se, assim, os cladódios analisados, visto que não foram encontradas na literatura referências ao tipo de cladódio que deve ser utilizado para análise de teor de nutrientes. Os cladódios foram lavados em água corrente e seccionados com auxílio de uma tesoura. Em seguida foram levados a estufa de circulação de ar forçada a $65^{\circ} \mathrm{C}$ até atingirem peso constante. Após a secagem dos cladódios, esses foram triturados e realizadas as análises de teores de nutrientes (N, P, K, Ca, Mg e S) de acordo com Malavolta, Vitti e Oliveira (1997).

Os dados foram submetidos à análise de variância (ANOVA), teste $\mathrm{F}$ e as médias comparadas pelo teste de Scott-Knott a 5\% de probabilidade.

\section{Resultados e Discussão}

Os tratamentos com $\mathrm{EB}+\mathrm{EG}+\mathrm{GB}$ e $\mathrm{EB}+\mathrm{EG}$ apresentaram maior produção por área em 2011, não diferindo estatisticamente entre si (Tabela 3), enquanto os sem adubação e os adubados apenas com granulado bioclástico apresentaram menores valores de produtividade, chegando o controle apresentar valor zero para essa característica.

Tabela 3. Médias da produtividade (PR), número de frutos produzidos por planta (NF), massa, rendimento de polpa, diâmetro longitudinal (DL), diâmetro transversal (DT), teor de sólidos solúveis (SS), acidez e relação SS/AT de frutos de pitaia vermelha adubadas com diferentes tipos de matéria orgânica em 2011(1).

\begin{tabular}{cccccccccc}
\hline Tratamentos & $\begin{array}{c}\text { PR } \\
\left(\mathrm{t} \mathrm{ha}^{-1}\right)\end{array}$ & NF & Massa $(\mathrm{g})$ & Polpa (\%) & DL (mm) & DT (mm) & $\begin{array}{c}\text { SS } \\
\left({ }^{\circ} \text { Brix }\right)\end{array}$ & $\begin{array}{c}\text { Acidez } \\
(\%)\end{array}$ & $\begin{array}{c}\text { Relação } \\
\text { SS/AT }\end{array}$ \\
\hline Controle & $0,00 \mathrm{c}$ & $0,25 \mathrm{e}$ & $86,32 \mathrm{~b}$ & $61,16 \mathrm{c}$ & $82,94 \mathrm{~b}$ & $66,49 \mathrm{~b}$ & $14,00 \mathrm{a}$ & $0,30 \mathrm{a}$ & $46,66 \mathrm{a}$ \\
EB & $4,20 \mathrm{~b}$ & $11,16 \mathrm{~d}$ & $167,86 \mathrm{a}$ & $64,57 \mathrm{~b}$ & $90,08 \mathrm{a}$ & $78,20 \mathrm{a}$ & $13,20 \mathrm{a}$ & $0,29 \mathrm{a}$ & $44,93 \mathrm{a}$ \\
EG & $3,40 \mathrm{~b}$ & $9,75 \mathrm{~d}$ & $168,05 \mathrm{a}$ & $63,54 \mathrm{~b}$ & $82,15 \mathrm{~b}$ & $73,54 \mathrm{a}$ & $13,86 \mathrm{a}$ & $0,30 \mathrm{a}$ & $46,18 \mathrm{a}$ \\
GB & $0,10 \mathrm{c}$ & $0,66 \mathrm{e}$ & $109,07 \mathrm{~b}$ & $54,75 \mathrm{~d}$ & $70,51 \mathrm{c}$ & $61,59 \mathrm{~b}$ & $14,43 \mathrm{a}$ & $0,29 \mathrm{a}$ & $48,69 \mathrm{a}$ \\
$\mathrm{EB}+\mathrm{EG}$ & $6,90 \mathrm{a}$ & $20,16 \mathrm{~b}$ & $173,06 \mathrm{a}$ & $71,10 \mathrm{a}$ & $92,81 \mathrm{a}$ & $81,89 \mathrm{a}$ & $14,40 \mathrm{a}$ & $0,31 \mathrm{a}$ & $45,72 \mathrm{a}$ \\
$\mathrm{EB}+\mathrm{GB}$ & $4,23 \mathrm{~b}$ & $14,00 \mathrm{c}$ & $159,65 \mathrm{a}$ & $60,58 \mathrm{c}$ & $86,66 \mathrm{a}$ & $77,89 \mathrm{a}$ & $15,06 \mathrm{a}$ & $0,31 \mathrm{a}$ & $47,74 \mathrm{a}$ \\
$\mathrm{EG}+\mathrm{GB}$ & $3,53 \mathrm{~b}$ & $9,66 \mathrm{~d}$ & $170,98 \mathrm{a}$ & $64,14 \mathrm{~b}$ & $90,91 \mathrm{a}$ & $71,73 \mathrm{a}$ & $14,73 \mathrm{a}$ & $0,33 \mathrm{a}$ & $44,79 \mathrm{a}$ \\
$\mathrm{EB}+\mathrm{EG}+\mathrm{GB}$ & $7,16 \mathrm{a}$ & $25,16 \mathrm{a}$ & $184,38 \mathrm{a}$ & $65,09 \mathrm{~b}$ & $91,21 \mathrm{a}$ & $82,33 \mathrm{a}$ & $14,33 \mathrm{a}$ & $0,33 \mathrm{a}$ & $44,05 \mathrm{a}$ \\
\hline $\mathrm{CV}(\%)$ & 13,2 & 13,8 & 9,0 & 3,9 & 4,8 & 5,7 & 5,4 & 7,4 & 8,1 \\
\hline
\end{tabular}

${ }^{(1)}$ Médias seguidas pela mesma letra minúscula na coluna não diferem entre si, pelo teste de Scott-Knott, a $5 \%$ de probabilidade. Fonte: Elaboração dos autores.

Observa-se que as plantas de pitaia vermelha adubadas com $\mathrm{EB}+\mathrm{EG}+\mathrm{GB}$ apresentaram valores de produção superiores às demais adubações, atingindo uma média de 25,2 frutos por planta (Tabela 3). As plantas nas quais foram aplicados EB+EG, também apresentaram altas produções sendo superadas apenas por aquelas que receberam $\mathrm{EB}+\mathrm{EG}+\mathrm{GB}$. O maior número de frutos pode ser atribuído ao maior volume de matéria orgânica (MO) aplicado nessas adubações, propiciando disponibilidade equilibrada de nutrientes para as plantas. Em contraste, as plantas sem adubação e com aplicação apenas de granulado bioclástico apresentaram menor número de frutos. 
Esses resultados possivelmente ocorreram devido aos diferentes teores de nutrientes na composição dos fertilizantes utilizados. Os estercos bovino e de galinha apresentam quantidades consideráveis de nitrogênio, enquanto que o granulado bioclástico não possui esse nutriente em sua composição. Além disso, as quantidades de fósforo e potássio do granulado bioclástico são muito inferiores às dos estercos utilizados.

Os principais elementos demandados pela pitaia e que devem ser fornecidos via fertilização são nitrogênio $(\mathrm{N})$, fósforo $(\mathrm{P})$ e potássio $(\mathrm{K})$ (HERNÁNDEZ, 2000). O nitrogênio estimula a emissão de raízes e brotos mais vigorosos, sendo mais requerido pela planta durante o crescimento vegetativo até o pré-florescimento da pitaia (LUDERS, 2004); o potássio promove aumento do diâmetro do caule (INTA, 1994), sendo um dos elementos mais requeridos especialmente por exercer as funções de translocação de carboidratos e regulação de abertura e fechamento de estômatos (MARSCHNER, 2005), segundo Castañer e Castañer (1980), o potássio exerce influência na formação dos tecidos e nos processos de respiração das plantas, aumentando os teores de açúcar, tamanho, peso e resistência ao manuseio dos frutos e também a flexibilidade dos tecidos e a resistência por parte das plantas às enfermidades; o fósforo apresenta maior demanda pela pitaia no início da formação dos frutos (INFANTE, 1996).

Caetano, Carvalho e Jasmim (2006) estudando os efeitos da adubação orgânica na cultura da figueira (Ficus carica L.) utilizando dois tratamentos (sem aplicação de esterco bovino e com a aplicação de $10 \mathrm{~kg}$ planta $^{-1}$ ), sendo todas as plantas adubadas de acordo com a recomendação básica para a cultura da figueira, obtiveram produção $13,9 \%$ maior no tratamento com adição de esterco bovino.

Em relação à massa do fruto, os tratamentos diferiram significativamente entre si, sendo que os frutos provenientes de plantas que não receberam adubação e daquelas adubadas apenas com granulado bioclástico apresentaram menores valores de massa quando comparados aos frutos dos demais tratamentos (Tabela 3). Esse resultado pode estar relacionado ao fato dos demais tratamentos terem recebido maiores doses de potássio $(\mathrm{K})$ e nitrogênio $(\mathrm{N})$, visto que o esterco de galinha e o esterco bovino apresentam maiores concentrações desses nutrientes, além disso, o K está intimamente relacionado a qualidade dos frutos. Contudo, em relação à porcentagem de polpa, no tratamento em que se aplicou EB+EG verificou-se frutos com maior porcentagem de polpa em relação aos demais tratamentos, com média de $71,1 \%$ de polpa. Os diâmetros longitudinal e transversal dos frutos provenientes de plantas sem adubação e fertilizadas apenas com granulado bioclástico foram inferiores aos dos frutos dos demais tratamentos.

Os frutos sem adubação ou apenas com a aplicação de granulado bioclástico apresentaram menor massa, rendimento de polpa e diâmetros longitudinal e transversal, possivelmente devido à falta de nutrientes fornecidos às plantas. Deduz-se que a aplicação apenas de granulado bioclástico não foi suficiente para garantir produções satisfatórias da pitaia com frutos de qualidade. Isso reflete a exigência nutricional da cultura, pois, provavelmente, a composição do granulado bioclástico não foi suficiente para promover maior massa, rendimento de polpa e diâmetro longitudinal e transversal dos frutos. Os baixos teores de $\mathrm{K}$ e $\mathrm{P}$ e a ausência de $\mathrm{N}$ na composição do granulado bioclástico possivelmente limitaram o desenvolvimento do fruto de pitaia nos tratamentos em que utilizou-se apenas esse fertilizante.

Cavalcante et al. (2011) estudando adubação orgânica em plantas de pitaia vermelha concluíram que o fornecimento de $20 \mathrm{~L}$ de esterco bovino por cova promoveu maior crescimento da parte aérea. De acordo com Chang, Chung e Tsai (2007), o esterco mineralizado propicia aumento do teor de húmus no solo, podendo elevar a capacidade de retenção de água dos solos arenosos, fornecendo nutrientes, possivelmente incrementando a atividade 
microbiana, resultando talvez em melhora do poder tampão do solo e do $\mathrm{pH}$.

Não houve diferença entre os tratamentos utilizados para as características teor de sólidos solúveis, acidez titulável e relação SS/AT (Tabela 3) de frutos de pitaia colhidos em 2011, indicando que essas características não são influenciadas pelo tipo de adubação utilizada. Os teores de sólidos solúveis, acidez e relação SS/AT encontrados por Yah et al. (2008) para a pitaia vermelha colhida 31 dias após antese são semelhantes aos obtidos no experimento sendo, respectivamente: 12,$6 ; 0,4$ e 33,5.

Em 2011, a adubação orgânica propiciou altos níveis de produção e produtividade para a pitaia, o que possivelmente, pode ser explicado pelo fato de o sistema radicular da planta ser superficial, por conseguinte, podendo absorver pequenos teores de nutrientes no solo (LE BELLEC; VAILLANT; INBERT, 2006), o que contribuiu para a formação de cultivos orgânicos, já que os nutrientes dos compostos orgânicos são disponibilizados lentamente. A utilização de compostos orgânicos e estercos de origem animal têm sido usados na Califórnia para a cultura da pitaia com sucesso (THOMSON, 2002).

Verifica-se que, em 2012, houve diferenças estatísticas entre os tratamentos para as características produtividade, número de frutos por planta, massa e rendimento de polpa (Tabela 4) e não foram observadas diferenças entre os tratamentos para as demais características avaliadas (Tabelas $4 \mathrm{e}$ 5). O número de frutos por planta e a produtividade da pitaia em 2012 foram maiores que em 2011 (Tabela 4), possivelmente devido as plantas ainda serem jovens apresentando produção crescente nos primeiros anos após o plantio. Não há relatos na literatura de que a pitaia apresente bianualidade. $\mathrm{O}$ tratamento que apresentou maior produtividade em 2012 foi com utilização de $\mathrm{EB}+\mathrm{EG}+\mathrm{GB}$, atingindo uma produtividade de 9,4 $\mathrm{t} \mathrm{ha}^{-1}$ o que representa um aumento de $31 \%$ em relação ao ano anterior. Esse resultado era esperado visto que as plantas de pitaia estão com quatro anos de idade e provavelmente ainda não atingiram estabilidade na produção. O tratamento onde se aplicou $\mathrm{EB}+\mathrm{EG}$ também apresentou alta produtividade $\left(7,0 \mathrm{t} \mathrm{ha}^{-1}\right)$, mas sem evolução quando comparado a 2011. O controle (sem adubação) e o tratamento com apenas o granulado bioclástico foram os que apresentaram menores produtividades em 2012.

Em relação à produção (número de frutos por planta) verificou-se a mesma tendência observada em 2011, no qual o tratamento adubado com $\mathrm{EB}+\mathrm{EG}+\mathrm{GB}$, foi o que apresentou maior número de frutos por planta (37,6 frutos/planta), o que representa um incremento de $50 \%$ em relação à 2011, seguido pelo tratamento adubado com $\mathrm{EB}+\mathrm{EG}$ (30,6 frutos/planta).

Para as características massa e rendimento de polpa, assim como em 2011, observa-se que os tratamentos adubados com EB+EG+GB e EB+EG foram os que apresentaram maiores valores para essas características respectivamente. O controle apresentou os valores mais baixos. A espessura de casca, diâmetro longitudinal e transversal dos frutos não foram influenciadas pelo tipo de adubação em 2012 (Tabela 4). Os resultados obtidos em 2012 se devem também ao fato de que os tratamentos que foram adubados com $\mathrm{EB}+\mathrm{EG}+\mathrm{GB}$ e $\mathrm{EB}+\mathrm{EG}$ receberam maiores quantidades de adubos e, consequentemente, atingiram maiores valores de produção. 
Tabela 4. Médias da produtividade (PR), número de frutos produzidos por planta (NF), massa, rendimento de polpa, espessura de casca (EC), diâmetro longitudinal (DL) e diâmetro transversal (DT) de frutos de pitaia vermelha adubadas com diferentes tipos de matéria orgânica em $2012^{(1)}$.

\begin{tabular}{cccccccc}
\hline \multirow{2}{*}{ Tratamentos } & PR $\left(\mathrm{t} \mathrm{ha}^{-1}\right)$ & NF & Massa $(\mathrm{g})$ & Polpa (\%) & EC (mm) & DL (mm) & DT (mm) \\
\hline Controle & $0,00 \mathrm{e}$ & $1,00 \mathrm{e}$ & $113,63 \mathrm{e}$ & $60,15 \mathrm{c}$ & $4,67 \mathrm{a}$ & $79,45 \mathrm{a}$ & $76,97 \mathrm{a}$ \\
EB & $4,20 \mathrm{c}$ & $25,83 \mathrm{c}$ & $217,49 \mathrm{c}$ & $66,78 \mathrm{a}$ & $4,70 \mathrm{a}$ & $85,33 \mathrm{a}$ & $77,61 \mathrm{a}$ \\
EG & $3,50 \mathrm{~d}$ & $21,06 \mathrm{~d}$ & $212,41 \mathrm{c}$ & $61,48 \mathrm{~b}$ & $4,57 \mathrm{a}$ & $83,91 \mathrm{a}$ & $79,26 \mathrm{a}$ \\
GB & $0,10 \mathrm{e}$ & $2,33 \mathrm{e}$ & $186,11 \mathrm{~d}$ & $55,69 \mathrm{~b}$ & $4,67 \mathrm{a}$ & $79,29 \mathrm{a}$ & $74,10 \mathrm{a}$ \\
EB+EG & $7,00 \mathrm{~b}$ & $30,60 \mathrm{~b}$ & $252,48 \mathrm{~b}$ & $71,14 \mathrm{a}$ & $4,61 \mathrm{a}$ & $87,54 \mathrm{a}$ & $84,83 \mathrm{a}$ \\
EB+GB & $4,73 \mathrm{c}$ & $17,53 \mathrm{~d}$ & $222,22 \mathrm{c}$ & $60,50 \mathrm{~b}$ & $4,59 \mathrm{a}$ & $86,45 \mathrm{a}$ & $79,40 \mathrm{a}$ \\
EG+GB & $3,40 \mathrm{~d}$ & $24,70 \mathrm{c}$ & $223,88 \mathrm{c}$ & $63,87 \mathrm{~b}$ & $4,60 \mathrm{a}$ & $88,15 \mathrm{a}$ & $80,03 \mathrm{a}$ \\
EB+EG+GB & $9,40 \mathrm{a}$ & $37,80 \mathrm{a}$ & $278,28 \mathrm{a}$ & $69,88 \mathrm{a}$ & $4,59 \mathrm{a}$ & $90,77 \mathrm{a}$ & $84,21 \mathrm{a}$ \\
\hline CV $(\%)$ & 13,6 & 15,2 & 6,4 & 3,4 & 4,4 & 5,4 & 5,1 \\
\hline
\end{tabular}

${ }^{(1)}$ Médias seguidas pela mesma letra minúscula na coluna não diferem entre si, pelo teste de Scott-Knott, a 5\% de probabilidade.

Fonte: Elaboração dos autores.

Damatto Júnior, Leonel e Pedroso (2005) trabalhando com adubação orgânica no cultivo do maracujazeiro-doce verificaram que a aplicação de $5,0 \mathrm{~kg}$ de esterco bovino por planta propiciou melhor qualidade, maior número de frutos e maior produção por planta. Leonel e Tecchio (2009) estudando o efeito da adubação orgânica na cultura da figueira encontraram que aplicação de esterco bovino aumentou a produtividade, produção e número de frutos, havendo poucas alterações nas dimensões dos mesmos. Após quatro anos de aplicação do esterco bovino, os mesmos autores obtiveram-se melhores resultados com 76 a $124 \%$ da dose de $\mathrm{N}$ recomendada para a cultura.

Os teores de sólidos solúveis (SS), acidez titulável, $\mathrm{pH}$ e relação SS/AT dos frutos de pitaia colhidos em 2012 não apresentaram diferenças significativas entre os tratamentos (Tabela 5). Esse mesmo resultado foi obtido em 2011, demonstrando que o tipo de adubação não interfere nas características químicas do fruto.

Tabela 5. Médias do teor de sólidos solúveis, $\mathrm{pH}$, acidez e relação SS/AT de frutos de pitaia vermelha adubadas com diferentes tipos de matéria orgânica em $2012^{(1)}$.

\begin{tabular}{ccccc}
\hline Tratamentos & SS ( ${ }^{\circ}$ Brix) & Acidez (\%) & pH & Relação SS/AT \\
\hline Controle & $12,73 \mathrm{a}$ & $0,28 \mathrm{a}$ & $4,43 \mathrm{a}$ & $45,89 \mathrm{a}$ \\
EB & $12,76 \mathrm{a}$ & $0,27 \mathrm{a}$ & $4,44 \mathrm{a}$ & $47,56 \mathrm{a}$ \\
EG & $12,70 \mathrm{a}$ & $0,26 \mathrm{a}$ & $4,64 \mathrm{a}$ & $48,21 \mathrm{a}$ \\
GB & $12,80 \mathrm{a}$ & $0,26 \mathrm{a}$ & $4,50 \mathrm{a}$ & $47,98 \mathrm{a}$ \\
EB + EG & $13,33 \mathrm{a}$ & $0,26 \mathrm{a}$ & $4,37 \mathrm{a}$ & $50,67 \mathrm{a}$ \\
EB + GB & $12,73 \mathrm{a}$ & $0,27 \mathrm{a}$ & $4,36 \mathrm{a}$ & $46,72 \mathrm{a}$ \\
EG + GB & $13,30 \mathrm{a}$ & $0,26 \mathrm{a}$ & $4,56 \mathrm{a}$ & $49,91 \mathrm{a}$ \\
EB + EG + GB & $13,00 \mathrm{a}$ & $0,27 \mathrm{a}$ & $4,40 \mathrm{a}$ & $47,57 \mathrm{a}$ \\
\hline CV $(\%)$ & 6,3 & 5,0 & 3,2 & 9,9 \\
\hline
\end{tabular}

${ }^{(1)}$ Médias seguidas pela mesma letra minúscula na coluna não diferem entre si, pelo teste de $\mathrm{F}$, a $5 \%$ de probabilidade.

Fonte: Elaboração dos autores. 
Damatto Júnior (2006) e Oliveira, Amaro Filho e Moura Filho (2006) trabalhando com bananeira 'Prata-anã' (Musa spp.) e meloeiro (Cucumis melo L.), respectivamente, adubada com diferentes doses de composto orgânico também não encontraram diferenças significativas para as características de qualidade dos frutos (textura, teor de sólidos solúveis, acidez titulável e $\mathrm{pH}$ ) entre os tratamentos analisados.

Os resultados obtidos nesse experimento podem ser relacionados aos teores de nutrientes encontrados nos cladódios da pitaia (Tabela 6).
Verificou-se que os teores de $\mathrm{N}$ e cálcio $(\mathrm{Ca})$ nos cladódios do controle foram inferiores aos encontrados nos demais tratamentos, o que indica que esses nutrientes podem ter limitado a produção deste tratamento. Os teores de $\mathrm{P}$ e magnésio $(\mathrm{Mg})$ nos cladódios do controle e das plantas adubadas apenas com granulado bioclástico foram inferiores aos demais tratamentos, deduzindo, que a ausência de adubação ou a adubação apenas com granulado bioclástico sem adição de esterco é limitante para a adequada nutrição das plantas de pitaia e, consequentemente, para sua produção.

Tabela 6. Teores médios de nutrientes $\left(\mathrm{g} \mathrm{kg}^{-1}\right)$ : nitrogênio $(\mathrm{N})$, fósforo $(\mathrm{P})$, potássio $(\mathrm{K})$, cálcio $(\mathrm{Ca})$, magnésio $(\mathrm{Mg})$ e enxofre (S) na matéria seca dos cladódios de pitaia vermelha em função das diferentes adubações ${ }^{(1)}$.

\begin{tabular}{ccccccc}
\hline Tratamentos & $\mathrm{N}$ & $\mathrm{P}$ & $\mathrm{K}$ & $\mathrm{Ca}$ & $\mathrm{Mg}$ & $\mathrm{S}$ \\
\hline Controle & $3,9 \mathrm{~b}$ & $2,7 \mathrm{~b}$ & $22,3 \mathrm{a}$ & $27,8 \mathrm{~b}$ & $4,2 \mathrm{c}$ & $0,5 \mathrm{a}$ \\
EB & $7,5 \mathrm{a}$ & $3,1 \mathrm{a}$ & $22,8 \mathrm{a}$ & $54,5 \mathrm{a}$ & $5,2 \mathrm{a}$ & $0,5 \mathrm{a}$ \\
EG & $7,6 \mathrm{a}$ & $3,8 \mathrm{a}$ & $22,9 \mathrm{a}$ & $64,6 \mathrm{a}$ & $5,0 \mathrm{a}$ & $0,5 \mathrm{a}$ \\
GB & $6,7 \mathrm{a}$ & $2,1 \mathrm{~b}$ & $21,8 \mathrm{a}$ & $51,1 \mathrm{a}$ & $4,6 \mathrm{~b}$ & $0,6 \mathrm{a}$ \\
EB + EG & $8,4 \mathrm{a}$ & $4,6 \mathrm{a}$ & $20,3 \mathrm{a}$ & $58,4 \mathrm{a}$ & $5,0 \mathrm{a}$ & $0,6 \mathrm{a}$ \\
EB + GB & $7,8 \mathrm{a}$ & $3,6 \mathrm{a}$ & $22,3 \mathrm{a}$ & $65,8 \mathrm{a}$ & $5,2 \mathrm{a}$ & $0,6 \mathrm{a}$ \\
EG + GB & $7,7 \mathrm{a}$ & $3,8 \mathrm{a}$ & $21,1 \mathrm{a}$ & $63,0 \mathrm{a}$ & $4,9 \mathrm{a}$ & $0,6 \mathrm{a}$ \\
EB $+\mathrm{EG}+\mathrm{GB}$ & $7,6 \mathrm{a}$ & $4,1 \mathrm{a}$ & $22,2 \mathrm{a}$ & $54,3 \mathrm{a}$ & $5,1 \mathrm{a}$ & $0,5 \mathrm{a}$ \\
\hline CV $(\%)$ & 15,1 & 15,1 & 17,0 & 20,0 & 4,0 & 15,2 \\
\hline
\end{tabular}

${ }^{(1)}$ Médias seguidas pela mesma letra minúscula na coluna não diferem entre si, pelo teste de Scott-Knott, a 5\% de probabilidade. Fonte: Elaboração dos autores.

Os teores médios de nutrientes encontrados no tratamento contendo $\mathrm{EB}+\mathrm{EG}+\mathrm{GB}(\mathrm{N}(7,6$ $\left.\mathrm{g} \mathrm{kg}^{-1}\right), \mathrm{P}\left(4,1 \mathrm{~g} \mathrm{~kg}^{-1}\right), \mathrm{K}\left(22,2 \mathrm{~g} \mathrm{~kg}^{-1}\right), \mathrm{Ca}(54,3 \mathrm{~g}$ $\left.\mathrm{kg}^{-1}\right), \operatorname{Mg}\left(5,1 \mathrm{~g} \mathrm{~kg}^{-1}\right)$ e $\left.\mathrm{S}\left(0,5 \mathrm{~g} \mathrm{~kg}^{-1}\right)\right)$ mostraramse satisfatórios para a produção de frutos de pitaia com adubação orgânica (Tabela 6). Moreira et al. (2012) avaliando o efeito da adubação orgânica na pitaia encontraram teores de $\mathrm{N}, \mathrm{P}$ e $\mathrm{K}$ diferentes dos obtidos no presente trabalho, com o nitrogênio variando de 11 a $16 \mathrm{~g} \mathrm{~kg}^{-1}$, fósforo de 1,9 a $3,3 \mathrm{~g} \mathrm{~kg}^{-1}$ e potássio de 23 a $26 \mathrm{~g} \mathrm{~kg}^{-1}$. Possivelmente esses resultados se devem a ausência de metodologia para coleta e avaliação de teores de nutrientes em pitaia o que dificulta a padronização dos cladódios retirados para análise foliar.

\section{Conclusão}

A aplicação de esterco bovino + esterco de galinha associado ao granulado bioclástico foi a melhor combinação resultando em maior produtividade. A adubação orgânica não influenciou as características químicas avaliadas. A adubação apenas com granulado bioclástico não foi suficiente para garantir produções satisfatórias da pitaia com frutos de qualidade.

\section{Agradecimentos}

Ao Conselho Nacional de Desenvolvimento Científico e Tecnológico e à Coordenação de Aperfeiçoamento de Pessoal de Nível Superior, 
pela concessão de bolsa de pós-doutorado, processo número 500242/2012-7.

\section{Referências}

ASSOCIATION OF OFFICAL ANALITICAL CHEMISTS - AOAC. Official methods of analysis of the association of official analitical chemists. $18^{\text {th }}$ ed. Washington: AOAC, 2007. $1750 \mathrm{p}$.

CAETANO, L. C. S.; CARVALHO, A. J. C.; JASMIM, J. M. Preliminary report on yield productivity and mineral composition of the fig trees as a function of boron and cattle manure fertilization in Brazil. Fruits, Paris, v. 61, n. 5, p. 341-349, 2006.

CASTAÑER, M. A.; CASTAÑER, J. A. Horticultura: guia prático. Lerida: Dilagro, 1980. 529 p.

CAVALCANTE, I. H. L.; MARTINS, A. B. G.; SILVA JÚNIOR, G. B.; ROCHA, L. F.; FALCÃO NETO, R.; CAVALCANTE, L. F. Adubação orgânica e intensidade luminosa no crescimento e desenvolvimento inicial da pitaya em Bom Jesus-PI. Revista Brasileira de Fruticultura, Jaboticabal, v. 33, n. 3, p. 970-983, 2011.

CHANG, E. H.; CHUNG, R. S.; TSAI, Y. H. Effect of different application rates of organic fertilizer on soil enzyme activity and microbial population. Soil Science \& Plant Nutrition, Tokyo, v. 53, n. 2, p. 132-140, 2007.

DAMATTO JÚNIOR, E. R. Influência da adubação com composto orgânico na qualidade de frutos de bananeira. Revista Científica Eletrônica de Agronomia, Garça, v. 5, n. 10, p. 1-7, 2006.

DAMATTO JÚNIOR, E. R.; LEONEL, S.; PEDROSO, C. J. Adubação orgânica na produção e qualidade de frutos de maracujá-doce. Revista Brasileira de Fruticultura, Jaboticabal, v. 27, n. 1, p. 188-190, 2005.

DIAS, G. T. M. Granulados bioclásticos - algas calcárias. Revista Brasileira de Geofísica, São Paulo, v. 18, n. 13, p. 307-318, 2000.

GALBIATTI, J. A.; GARCIA, A.; SILVA, M. L. D.; MASTROCOLA, M. A.; CALDEIRA, D. S. A. Efeitos de diferentes doses e épocas de aplicação de efluente de biodigestor e da adubação mineral em feijoeiro-comum (Phaseolus vulgaris L.) submetido a duas lâminas de água por meio de irrigação por sulco. Científica, Jaboticabal, v. 24, n. 1, p. 63-74, 1996.

HAFLE, O. M.; SANTOS, V. A.; RAMOS, J. D.; CRUZ, M. C. M.; MELO, P. C. Produção de mudas de mamoeiro utilizando Bokashi e Lithothamnium. Revista Brasileira de Fruticultura, Jaboticabal, v. 31, n. 1, p. 245-251, 2009.
HERNÁNDEZ, Y. D. O. Hacia el conocimiento y la conservación de la pitahaya. Oaxaca: Ipn-Sibej-ConacytFmcn, 2000. 124 p.

INFANTE, G. S. El cultivo de la pitahaya: experiencias en Colombia. In: CASTILlO, M.; CÁlIX, H. (Ed.). Memoria del primer curso teórico-práctico sobre el cultivo de la pitahaya. Quintana: Universidad de Quintana Roo, 1996. p. 17-31.

INSTITUTO NICARAGUENSE DE TECNOLOGÍA AGROPECUÁRIA - INTA. Guía técnica para la produción de pitahaya. San Marcos: Carazo, 1994. 52 p.

LE BELLEC, F.; VAILLANT, F.; INBERT, E. Pitahaya (Hylocereus spp.): a new crop, a market with a future. Fruits, Paris, v. 61, n. 4, p. 237-250, 2006.

LEONEL, S.; TECCHIO, M. A. Cattle manure fertilization increases fig yield. Scientia Agricola, Piracicaba, v. 66, n. 6, p. 806-811, 2009.

LUDERS, L. The pitaya or dragon fruit (Hylocereus undatus). Darwin: University of Darwin, 2004. 5 p.

MALAVOLTA, E.; VITTI, G. C.; OLIVEIRA, S. A. Avaliação do estado nutricional de plantas; princípios e aplicações. Piracicaba: Potafós, 1997. 391 p.

MARSCHNER, H. Mineral nutrition of higher plants. $6^{\text {th }}$ ed. London: Academic, 2005. 889 p.

MARQUES, V. B. Germinação, fenologia e estimativa do custo de produção da pitaia [Hylocereus undatus (Haw.) Britton \& Rose]. 2010. Tese (Doutorado em Agronomia) - Universidade Federal de Lavras, Lavras.

MELO, P. C.; FURTINI NETO, A. E. Avaliação do Lithothamnium como corretivo da acidez do solo e fonte de nutrientes para o feijoeiro. Ciência e Agrotecnologia, Lavras, v. 27, n. 3, p. 508-519, 2003.

MOREIRA, A.; FAGERIA, N. K.; SOUZA G. B.; FREITAS, A. R. Production, nutritional status and chemical properties of soil with addition of cattle manure, reactive natural phosphate and biotite schiste in Massai cultivar. Revista Brasileira de Zootecnia, Viçosa, MG, v. 39, n. 9, p. 1883-1888, 2010.

MOREIRA, R. A.; RAMOS, J. D.; ARAÚJO, N. A.; MARQUES, V. B.; MELO, P. C. Produtividade e teores de nutrientes em cladódios de pitaia vermelha utilizandose adubação orgânica e granulado bioclástico. Revista Brasileira de Ciências Agrárias, Recife, v. 7, p. 714-719, 2012. Suplemento.

OLIVEIRA, A. M. G.; CALDAS, R. C. Produção do mamoeiro em função de adubação com nitrogênio fósforo e potássio. Revista Brasileira de Fruticultura, Jaboticabal, v. 26, n. 1, p. 160-163, 2004. 
OLIVEIRA, A. N. P.; OLIVEIRA, A. P.; LEONARDO, F. A. P.; CRUZ, I. S.; SILVA, D. F. Yield of gherkin in response to doses of bovine manure. Horticultura Brasileira, Brasília, v. 27, n. 1, p. 100-102, 2009.

OLIVEIRA, F. J. M.; AMARO FILHO, J.; MOURA FILHO, E. R. Efeito da adubação orgânica sobre a qualidade de frutos de meloeiro (Cucumis melo L.). Revista Verde, Mossoró, v. 1, n. 2, p. 81-85, 2006.

SANTOS, R. H. S.; MENDONÇA, E. S. Agricultura natural, orgânica, biodinâmica e agroecológica. Informe Agropecuário, Belo Horizonte, v. 22, n. 212, p. 5-8, 2000.
SILVEIRA, J.; GALESKAS, H.; TAPETTI, R.; LOURENCINI, I. Quem é o consumidor brasileiro de frutas e hortaliças? Hortifruti Brasil, Piracicaba, v. 10, n. 103, p. 8-22, 2011.

THOMSON, P. Pitahaya (Hylocereus species): a promising new fruit crop for Southern California. Bonsall: Bonsall Publications, 2002. 72 p.

YAH, A. R. C.; PEREIRA, S. S.; VELOZ, C. S.; SAÑUDO, R. B.; DUCH, E. S. Cambios físicos, químicos y sensoriales en frutos de pitahaya (Hylocereus undatus) durante su desarrollo. Revista Fitotecnia Mexicana, Chapingo, v. 31, n. 1, p. 1-5, 2008. 
